\title{
Surrogate human sensor for human skin surface temperature measurement in evaluating the impacts of thermal behaviour at outdoor environment
}

\author{
Yee Yong Lee ${ }^{\mathrm{a}, *}$, Mohd Fadhil Md Din ${ }^{\mathrm{b}}$, Zainura Zainon Noor ${ }^{\mathrm{b}}$, Kenzo Iwao ${ }^{\mathrm{c}}$, Shazwin Mat Taib ${ }^{\mathrm{b}}$, \\ Lakhveer Singh $^{\mathrm{d}}$, Nur Hafizah Abd Khalid ${ }^{\mathrm{e}}$, Nickholas Anting ${ }^{\mathrm{a}}$, Eeydzah Aminudin ${ }^{\mathrm{e}}$ \\ a Jamilus Research Center, Faculty of Civil and Environmental Engineering, Universiti Tun Hussein Onn Malaysia, 86400 Parit Raja, Batu Pahat, Johor, Malaysia \\ ${ }^{\mathrm{b}}$ Center of Environmental Sustainability and Water Security (IPASA), Research Institute of Sustainable Environment (RISE), Universiti Teknologi Malaysia, 81310 UTM \\ Johor Bahru, Malaysia \\ ${ }^{\mathrm{c}}$ Nagoya Institute of Technology, Incubation Center, Building 2, Room 622B, Gokiso-cho, Showa-ku, Nagoya-city, Aichi-Pref. 466-8555, Japan \\ d Faculty of Industrial Sciences \& Technology, Universiti Malaysia Pahang (UMP), Lebuhraya Tun Razak, 26300 Pahang, Malaysia \\ e Department of Structure and Materials, Faculty of Civil Engineering, Universiti Teknologi Malaysia, 81310 Johor Bahru, Johor, Malaysia
}

\section{A R T I C L E I N F O}

\section{Keywords:}

Thermal environment

Ergonomics

Thermal comfort

Human skin surface measurement

Outdoor environment

Surrogate Human Sensor

Thermal behaviour

\begin{abstract}
A B S T R A C T
The world is experiencing high rates of urbanisation and it has slowly become an alarming social process, especially in developing countries. This has demanded an urgent investigation on human thermal comfort, especially in tropical climates. In this study, a surrogate human sensor (SHS) was developed to establish a linkage between human skin surface and SHS with the surrounding environments. Black plastic corrugated cardboard was used in the SHS fabrication as its thermal conductivity was close to the thermal conductivity and emissivity of the human epidermal skin layer. The SHS was designed to correlate with human skin surface temperature and a regression model was developed. The regression equation was obtained for the human skin temperature prediction $\left(\mathrm{T}_{\mathrm{h}}\right)$ by using SHS. Statistical analysis of the ANOVA $(\mathrm{F}=13,700 ; \rho<0.05)$ was significantly tested to show its reliability. The predicted and measured human skin temperature was compared and the results revealed that both temperature variations was found in range $\pm 0.5^{\circ} \mathrm{C}$ in temperature differences. The advantages of SHS as the sensor for the impact of thermal behaviour can be identified by observing the temperature difference as it can directly reflects the influences from the surrounding outdoor environment. Although it is proven valid statistically, however, SHS is only relevant as an initial indicator to investigate the impacts of thermal behaviour and discomfort level. It can further used to measure human thermal comfort by correlating surrounding environment condition with comfort sensation through SHS regression model.
\end{abstract}

\section{Introduction}

Thermal sensation was investigated initially by Fanger [1-2] in an indoor environment who focused on the correlation of the physical parameters, physiological parameters and human perception towards surrounded environment. His group introduced comfort index of Predicted Mean Vote (PMV) and Predicted Percentage of Dissatisfied (PPD), leading to other related indicators such as Heat Stress Indices [3]. Throughout the research development, adaptive thermal comfort has been concentrated [4-7]. In warm climates, it has been found behavioural adaptation is one of the overestimation of PMV index [8-9].

Outdoor thermal environment of urban spaces plays a great role on the quality of life in a city. It directly affects people's comfort or behaviour and usage of outdoor spaces. In the path of investigation, the thermal behaviour of urban environment has been documented by various researchers, both in anthropogenic factors [10-12] and 'albedo' effects of surface material [13-15] as well as the urban arrangement $[16,17]$. Evaluating the thermal environment, particularly when it concerns the human body and health, is important so as to improve our way of living. The level of human thermal comfort is highly dependent on the urban microclimate environment whereby thermal sensations and comfort in indoor and outdoor environments are expected to decrease along with changes in climatic conditions [18,19]. The significant consequences from the impacts of thermal behaviour of the surrounding built-up environment is known as Urban heat Island (UHI) phenomenon which may further affect human thermal comfort especially in urban outdoor conditions. Various new strategies for improving the state of ambient comfort have been outlined as they directly influence our energy consumption efficiency, which is mainly linked to sustainable development, and it also correlates closely with

\footnotetext{
* Corresponding author

E-mail address: yylee@uthm.edu.my (Y.Y. Lee).
} 